Revue Française de Civilisation Britannique

\title{
Introduction : Les Enjeux de l'interdisciplinarité en civilisation britannique
}

Introduction

Romain Garbaye et Gilles Leydier

\section{(2) OpenEdition}

Journals

Édition électronique

URL : http://journals.openedition.org/rfcb/2738

DOI : $10.4000 /$ rfcb. 2738

ISSN : 2429-4373

Éditeur

CRECIB - Centre de recherche et d'études en civilisation britannique

Référence électronique

Romain Garbaye et Gilles Leydier, «Introduction : Les Enjeux de l'interdisciplinarité en civilisation britannique », Revue Française de Civilisation Britannique [En ligne], XXIV-1 | 2019, mis en ligne le 22 mars 2019, consulté le 01 mai 2019. URL : http://journals.openedition.org/rfcb/2738 ; DOI : 10.4000/ rfcb. 2738

Ce document a été généré automatiquement le 1 mai 2019.

\section{(i)

Revue française de civilisation britannique est mis à disposition selon les termes de la licence Creative Commons Attribution - Pas d'Utilisation Commerciale - Pas de Modification 4.0 International. 


\title{
Introduction : Les Enjeux de l'interdisciplinarité en civilisation britannique
}

\author{
Introduction
}

Romain Garbaye et Gilles Leydier

1 La période actuelle est marquée par un intérêt croissant pour les questions d'approches disciplinaires, d'interdisciplinarité et de méthodologie dans les divers champs des études anglophones : réflexion sur les différents types de «studies», sur la notion " d'aires culturelles ", ou sur l'interdisciplinarité. A partir d'un atelier du Centre de Recherches et d'Etudes en Civilisation Britannique (CRECIB) qui s'est tenu en 2017 au congrès annuel de la SAES à Reims, la Revue Française de Civilisation Britannique propose ici des pistes de réflexion sur les débats théoriques et méthodologiques actuels en civilisation britannique pour contribuer à la réflexion collective sur l'évolution du champ. Comment étudier la Grande-Bretagne aujourd'hui en utilisant les perspectives et les méthodes de l'histoire et des sciences sociales (sociologie, science politique, anthropologie....) Comment penser la civilisation en référence aux autres domaines de l'anglistique, comme la littérature, la linguistique, le cinéma, etc. Comment la notion « d'aire culturelle » peut-elle aider à articuler civilisation et interdisciplinarité ? Enfin, quel usage faire de la comparaison en civilisation britannique?

2 Cornelius Crowley ouvre ce numéro avec un regard rétrospectif sur l'évolution de la civilisation britannique telle qu'elle se pratique dans l'université française dans les trente dernières années. Pour ce faire, il se base sur les apports conceptuels de la sociologie de Pierre Bourdieu à l'étude des systèmes éducatifs en général et à ses analyses de l'enseignement supérieur en particulier. Ce retour est la condition d'une réflexion critique sur les modalités de construction historique de ce champ permettant l'identification des modalités d'organisation, de régulation, concomitante d'une autonomisation de ce champ et d'une augmentation « des droits d'entrée ». Ceci implique que la spécialisation toujours plus élevée de ce champ requiert de ses participants la mise en adéquation de leur objet d'étude avec des méthodologies appropriées. L'histoire de la 
civilisation britannique comme discipline fait ainsi écho à celle de nombreuses autres disciplines, pour lesquelles une autonomisation toujours plus marquée au sein d'un champ universitaire se double d'une logique de spécialisation.

3 Sam Coombes apporte à la réflexion la perspective d'un chercheur en études postcoloniales situé dans le contexte universitaire britannique. De ce point de vue, la question de l'interdisciplinarité dans les études de civilisation britannique telles qu'elles sont pratiquées en France prend une tournure particulière, qui lui permet d'apporter des éclairages originaux. Faisant écho aux réflexions de Cornélius Crowley sur l'impact du processus de Bologne en France, il insiste sur le cadre institutionnel de la recherche britannique en sciences humaines et sociales, pour montrer comment l'interdisciplinarité est souvent comprise comme un objectif à atteindre fixé par les organismes financeurs de la recherche pour les candidats aux appels à projets, dans un contexte de concurrence et de marchandisation de l'enseignement supérieur qui pousse au décloisonnement disciplinaire et à l'affichage de l'innovation, compris comme des conditions d'attractivité et de compétitivité. Plus fondamentalement, cependant, l'interdisciplinarité n'a pas attendu l'avènement de la logique de la recherche par projet pour s'imposer comme un fait dans les sciences humaines et sociales, dont les objets de recherche complexes, qui, par leur construction même, se trouvent souvent à la croisée de plusieurs domaines d'études et s'appréhendent avec des méthodologies composites. L'exemple des études théâtrales donné par Sam Coombes est évocateur de nombreux autres domaines des sciences humaines et sociales concernés par la civilisation britannique, des études de productions culturelles comme l'iconographie, le cinéma ou la musique populaire, mais aussi les discours politiques ou les représentations des identités nationales.

4 L'auteur se penche aussi sur la question de la comparaison - qui n'est pas nécessaire à l'interdisciplinarité, mais s'y prête particulièrement bien- pour montrer tout son intérêt pour des études définies en référence à une aire appréhendée en termes de singularité linguistique, historique, géographique et/ou culturelle, comme l'est la civilisation britannique. Il propose pour ce faire un détour fécond par l'œuvre du penseur postcolonial francophone Edouard Glissant, qui pense la langue créole de sa terre natale de Martinique dans son rapport à la langue et à la culture de la métropole coloniale. C'est ainsi que se crée un objet d'étude défini en termes interculturels, qui ne peut donc être compris que dans sa relation à d'autres influences et apports, tout en revendiquant sa singularité. Dans cette perspective, l'objet d'étude ne peut être réifié par référence à une langue ou une culture canonique et figée, ni réduit à son appréhension par des outils méthodologiques de sciences sociales à vocation universelle et donc appliqués de manière interchangeable à toutes les aires culturelles.

5 Cette question de la comparaison s'impose comme un enjeu méthodologique d'un nombre croissant de travaux en civilisation britannique dans la période récente, en même temps que l'interdisciplinarité, poursuivant la voie tracée par la génération précédente. Il n'est pas anodin de constater que certains des chercheurs les plus influents de cette génération fondatrice des années 1970 et 1980, comme Roland Marx ou Monica Charlot, se remarquaient déjà pour leur formation interdisciplinaire (avec les apports de l'histoire ou de la science politique). A travers la méthode comparée, et le comparatisme qui place cette méthode au cœur de la démarche du chercheur, les civilisationnistes actuels cherchent à éprouver de manière fine et circonstanciée la singularité de la GrandeBretagne au regard d'autres pays qui peuvent être construits par le regard du chercheur comme des «cas » comparables. C'est ce que montre Vincent Latour à travers l'exemple 
des études politiques contemporaines, où des approches dérivées de la politique comparée ou de la sociologie politique permettent de s'interroger sur la "britannicité » d'évolutions politiques comme l'avènement des lois contre la discrimination raciale et le multiculturalisme politique. C'est en déployant un regard comparatif trans-Manche que le chercheur peut se livrer à l'exercice du «jeu de miroir» et identifier des éléments explicatifs du contraste franco-britannique dans ce domaine, comme l'influence du mouvement des droits civiques américains en Grande-Bretagne au moment des premières lois contre la discrimination raciale dans les années 1960.

6 Susan Ball s'interroge sur la place occupée par la notion d'espace et les nouveaux paradigmes de la géographie humaine au sein de la recherche française en civilisation britannique contemporaine. En effet si un spatial turn a bien été identifié dans le domaine des sciences humaines et sociales au cours des dernières années, il a aussi dans une certaine mesure influencé les études en civilisation britannique, par ailleurs imprégnées de problématiques culturelles et sociales. A partir d'un corpus d'articles publiés dans la Revue Française de Civilisation Britannique au cours des deux dernières décennies, Susan Ball analyse comment les chercheurs anglicistes ont intégré les problématiques liées à la localisation et à l'espace, sans pour autant mettre à l'écart les dimensions temporelle et sociétale de leur objet d'études. Une dialectique socio-spatiale féconde a ainsi irrigué la recherche, et le développement d'un «imaginaire géographique » chez les chercheurs civilisationnistes a contribué à positionner ces derniers au cœur des enjeux liés à l'interdisciplinarité dans le domaine des sciences humaines et sociales.

7 Vanessa Boullet s'intéresse à la place occupée par la "civilisation économique » au sein des études de civilisation contemporaine sur les pays anglophones. A partir de l'exemple de sa propre recherche portant sur les politiques économiques menées par les gouvernements successifs de la République d'Irlande, elle s'interroge sur les interactions disciplinaires entre les différents domaines constitués par l'économie politique, l'histoire politique, les Irish studies ou les Development studies. Selon elle, la civilisation économique offre le cadre à la fois conceptuel et pratique d'un nécessaire décloisonnement disciplinaire, permettant ainsi la prise en compte d'un contexte socio-politico-culturel large pour une compréhension approfondie de phénomènes économiques territorialisés et situés dans le temps. Si l'ancrage disciplinaire est nécessaire à la formation initiale du chercheur, et à la structuration de sa réflexion, la recherche d'une interaction féconde, à la fois pluri et interdisciplinaire, loin de remettre en cause les fondements épistémologiques propres à chaque discipline scientifique, constitue le prolongement indispensable de la démarche du chercheur civilisationniste, dans la perspective appréhender la réalité complexe et hybride d'un monde interconnecté et globalisé.

8 Spécialiste du Commonwealth, Mélanie Torrent s'attache à monter comment le dialogue interdisciplinaire a permis au fil des années de renouveler l'analyse de cet objet de recherches singulier. Espace géopolitique original, acteur-clé du multilatéralisme sur la scène mondiale, lieu de focalisation d'imaginaires pluriels, le Commonwealth et son histoire constituent pour les chercheurs un double défi nécessitant le décloisonnement et l'interaction entre spécialistes des différentes disciplines en sciences humaines et sociales et d'aires culturelles très diverses. Des problématiques comme celles des migrations, du transnationalisme, de la citoyenneté, de la globalisation, du soft power ou des diplomaties culturelles ont ainsi pu être renouvelées grâce à ces approches transversales abordant le Commonwealth comme un creuset unique, et un prisme d'analyse critique pertinent pour l'histoire du monde contemporain. Le dialogue interdisciplinaire entre chercheurs a 
permis non seulement d'enrichir la connaissance sur le Commonwealth, mais aussi de maintenir une forme d'équilibre entre ses différents lieux d'action et de mémoire, et entre ses représentations changeantes comme idée, pratique ou institution, en proposant une approche des relations internationales renouvelée et exposée dans toute sa complexité.

9 Pour conclure ce numéro, Jean-Paul Revauger revient fort utilement sur l'origine et le développement de la discipline « civilisation » au sein du champ des études anglophones de l'Université française depuis les années 1970. La distanciation vis-à-vis des autres composantes de l'« anglistique », les débats terminologiques autour du nom fondant la discipline, les interactions avec les sciences humaines et sociales, la relation avec les cultural studies, les dynamiques collectives de recherche, la place et l'intérêt du comparatisme, ou l'enjeu de l'interdisciplinarité sont autant de thèmes récurrents abordés dans cet entretien.

10 Près de cinq décennies après leur émergence au sein des départements d'anglais, les civilisationnistes continuent ainsi de revendiquer la légitimité de leur démarche scientifique originale, à la fois inclusive et transversale, qui, combinée à un rapport particulier à la langue, produit un regard unique et cohérent sur l'aire culturelle britannique ${ }^{1}$.

11 Ce numéro a été mis en page et préparé pour la publication, en utilisant le logiciel LODEL, par John Mullen.

\section{NOTES}

1. Gilles Leydier, "The Jack of All Trades, the Master of One, in G. Leydier (ed.), «La civilisation: Objet, enjeux, méthodes ", Revue Babel n9, 2004, Université de Toulon, pp. 7/20 